\title{
Diagnostic and therapeutic problems in nystagmus
}

\author{
KENNETH WYBAR \\ London
}

In all forms of dysfunction of ocular motility the ultimate aim in obtaining an under standing of the nature of the condition is to try and provide some form of treatment. This has been achieved in most types of strabismus with the considerable advances which have been made in overcoming the sensory and motor complications of the squint, but few sucN advances have been made in the treatment of nystagmus, and it is important, therefore, iq any symposium which is concerned with the use of prisms in the treatment of disorders of ocular motility, to take due regard of their role as one of the therapeutic measures im nystagmus even if this proves to be of very limited value.

There is, however, a fundamental difference between the problems of squint and those o nystagmus occurring in young children, because squint is most commonly an isolated event whereas nystagmus is usually a manifestation of some underlying disorder, even in the so called "idiopathic" cases. It follows that all cases of nystagmus must be assessed carefull before embarking on a line of treatment which is concerned with the nystagmus alone. is the purpose of this paper to discuss the nature of 120 cases of nystagmus which have bee $\mathbb{D}$ examined recently at the Hospital for Sick Children, Great Ormond Street, and a $\vec{\Phi}$ Moorfields Eye Hospital, High Holborn, in children under the age of 6 years, and to indicate the possible merits and limitations of treatment of cases of nystagmus with prismo

\section{CLA S S I FI CA T T O N}

\section{Idiopathic nystagmus}

In this series 45 of the 120 cases are regarded as belonging to the "idiopathic" ? category on the basis of an absence of any precise neurological (including vestibular) o⿱ ocular defect which appears to be sufficient to account for the nystagmus. It is obvious however, that cases are placed in this category simply because of a failure to detect the presence of some underlying defect, and it seems likely that many such cases are of neurof్ logical origin.

Sometimes there is a family history of the condition so that it is the result of a genetio factor which exhibits a varied form of inheritance; regular or irregular, dominant of recessive, sex-linked or autosomal. In most cases, however, there is no genetic basis and it seems likely that the causative lesion lies in the complex nervous mechanisms which are concerned in the centring of the eyes - the paramedian zone of the diencephalon, th brain-stem, the cerebellum, and the motor centres in the cerebral hemispheres. In thi⿺ way the nystagmus may be regarded to some extent as an exaggeration of the fine persisten $\bar{R}$

Read at the European (ouncil for Strabismological Studies in Amsterdam, on June 18, 1968, in a Symposium on Prismotherap 
movements of the eyes (slow-motion random drifts, rapid impulsive saccades which correct the position of the foveal image after the random drifts, and fine tremors) which occur during "steady" fixation and which are essential to the maintenance of the perception of the retinal image.

It follows that the usual term of "idiopathic congenital nystagmus" for such cases is somewhat misleading, because few cases have a truly congenital origin, hence the use simply of the term "idiopathic" nystagmus in this discussion.

There are two special forms of nystagmus which may be regarded as "idiopathic" or "congenital"- fixation nystagmus and latent nystagmus.

\section{FIXATION NYSTAGMUS}

There were five cases in this series of 45 idiopathic cases. The nystagmus is obvious only on attempted visual fixation, and it seems likely that this is essentially an exaggeration of the increase in nystagmus which is a common feature in the usual cases of idiopathic nystagmus during visual fixation; this increase in the nystagmus during fixation is also a feature of some forms of ocular nystagmus.

\section{LATENT NYSTAGMUS}

There were seven cases in this series of 45 idiopathic cases. The nystagmus is obvious only on a complete or partial dissociation of the eyes, but this does not imply that it is induced necessarily as the result of a breakdown in binocular single vision because it occurs most frequently in association with a manifest squint. It seems, therefore, that the nystagmus is maintained in a latent state provided the retina of each eye is receiving more or less similar visual stimuli even although the stimulus is appreciated in different ways by the two eyes because of the absence of binocular function.

It is obviously important in all cases of idiopathic nystagmus to carry out careful neurological and ocular examinations in order to exclude any underlying cause. It is sometimes necessary also to carry out electrodiagnostic tests, particularly the electroretinogram (ERG) but sometimes also the recording of visually evoked cortical responses (VER), in order to determine the potential visual function. Eight of the 45 cases of idiopathic nystagmus were regarded initially as having extremely poor vision (an appreciation only of light or dark), or even as being completely blind, because of the absence of any apparent visual interest in the early months of life; this was refuted by electrodiagnostic tests, so that it was possible to reassure the parents that the long-term visual prognosis was reasonably good, particularly for close reading. Conversely, three which were regarded initially as simply cases of idiopathic nystagmus were later shown by electrodiagnostic tests to be cases of tapeto-retinal degeneration.

It is common for nystagmus of the idiopathic type to vary in extent in different positions of gaze; an extreme example is the nystagmus which is of the jerky type on either side of a neutral point at which the eyes are free from nystagmus and at which the visual acuity is normal. Usually this neutral point is merely a position in which the nystagmus is less marked, and it may be the straight-ahead position or at any degree of eccentricity, so that an abnormal head posture is sometimes induced to make use of the neutral point with the eyes directed straight-ahead. The adoption of an abnormal head posture occurred in only three cases of the 45 idiopathic cases. 


\section{Neurological nystagmus}

In this series 43 of the 120 cases of nystagmus are regarded as neurological in originô Sometimes the mode of production of the nystagmus was clear-cut when there was evidence on investigation of a precise disorder involving the afferent visual pathway or some com? ponent of the efferent motor pathway which is concerned with the activity and co-ordina tion of the extrinsic ocular muscles. These include a craniopharyngioma (2), a suprasella $\overline{\frac{}{p}}$. astrocytoma (I), a papilloma of the choroidal plexus (I), a chiasmal glioma (I), a menin $\mathbb{8}$ gioma (I), a cerebellar astrocytoma (I), a cerebellar medulloblastoma (I), a brain-stents glioma (I), a developmental anomaly of the brain (I), a partial cerebral and cerebella5 atrophy (I), hydrocephalus (6) with some degree of optic atrophy in four and a paresis of cranial nerve VI in one, evidence of cerebral palsy (7), evidence of severe mental retarda tion (4) one with an amino-aciduria, evidence of brain damage as the result of neonata $\$$ aroxia (5), and optic atrophy as the result of a cranio-facial dysostosis ( 1 ).

There were also nine cases of optic atrophy of unknown origin among the 43 "neurot logical" cases in which there was no definite evidence of any other neurological or ocula N disorder. Sometimes optic atrophy in early childhood may be of an inherited nature: $\vec{b}$ recessive condition of congenital or infantile origin in which both the parents are health $\overrightarrow{\underline{L}}$ carriers (Waardenburg, I 957), a dominant condition of infantile origin (Kjer, I959), or $\underset{8}{\mathrm{P}}$ condition occurring in male or female children in families showing the typical features of Leber's optic atrophy (Wilson, 1967). It is, of course, possible that optic atrophy occurso in infancy as the result of a period of anoxia during birth (for example, following a prema ture separation of the placenta) or during the neonatal period. It is evident experiment ally that the cerebral structures are somewhat resistant to the effects of anoxia (Windle I950; Ford, I952), but it is doubtful if this applies to man because of the necrosis whic occurs in the white matter following anoxia; this is illustrated by the case of a 2-year-olक्षे child who developed an advanced bilateral optic atrophy following a prolonged period of profound anoxia after general anaesthesia in the absence of any other contributory cause

\section{Ocular nystagmus}

In this series 22 of the 120 cases of nystagmus are regarded as ocular in origin. These include cataract (3), a persistent hyperplastic vitreous (I), a retinal dysplasia (I), a retro lental fibroplasia (2), a tapeto-retinal degeneration (3), a cone dysfunction syndrome (i) $\frac{0}{3}$ an optic disc hypoplasia ( $\mathrm{I}$ ), albinism (5) which was only partial in three, aniridia (2), and్ర amblyopia of disuse in association with squint (3).

It is not strictly relevant to the study of nystagmus but it is significant that cases of retrolental fibro․ㅡㄹ plasia (the retinopathy of prematurity) still occur sporadically despite the knowledge that they arf produced by high oxygen therapy. This is due in part to the necessity of administering oxyge when there is a significant degree of anoxia in the neonatal period, particularly in the prematur尺 infant, in order to save life and to reduce the incidence and extent of brain damage, and also in part to the difficulty in assessing the amount of oxygen which is liable to affect the retina adversely. The percentage of oxygen in the atmosphere tends to be an inaccurate criterion, and it is necessary to estimate the arterial blood oxygen levels at frequent intervals, or to make an indirect assessment b@

repeated examinations of the fundi for evidence of retinal vasospasm.
There are ten other cases of nystagmus which are not included in the three main categories four which are probably of the idiopathic type despite the presence of a doubtful degree o\$ optic atrophy in three cases and a partial cataract in one eye of one case; three in which th ocular movement appeared to be essentially of a nystagmoid nature; and two which showeck 
peculiar ocular movements which are not strictly those of nystagmus-one case of ocular dysmetria in a brain-stem lesion of unknown origin, and one case of ocular myoclonus in association with a generalized myoclonus following an encephalitis with brain-stem involvement.

TREATMENT

The treatment of nystagmus may be applied directly to the abnormal movements of the eyes or indirectly to the cause of the nystagmus.

\section{Indirect methods}

These are obviously of great importance because they are concerned with the condition which is the cause of the nystagmus; there is certainly no value in treating the eyes when the nystagmus is due to some progressive neurological disorder. The diagnosis and treatment of these underlying disorders, however, are not part of the scope of this discussion.

\section{Direct methods}

These include the use of prisms and surgical procedures.

\section{Prismotherapy}

This may be applied to the treatment of nystagmus in two ways.

(1) When the nystagmus is significantly diminished (or sometimes even absent) in a certain position of conjugate lateral gaze with a consequent enhancement of the visual acuity, and particularly when such a position is sought actively by the patient by the adoption of an abnormal head posture, prisms of appropriate and equal strengths may be placed in front of each eye (base-in in front of one eye and base-out in front of the other eye) in order to maintain the eyes in the advantageous position of conjugate gaze without the necessity of adopting the abnormal head posture. For example, when the nystagmus is least in a position of laevoversion, so that the abnormal head posture consists of an equivalent degree of face turning to the right, a prism of appropriate strength is placed base-out in front of the right eye and a prism of equal strength is placed base-in in front of the left eye in order to retain the eyes in the position of laevoversion and yet permit the binocular fixation of a straight-ahead object in the absence of an abnormal head posture.

It is interesting, however, that in this series of 120 cases of nystagmus there are only three (all in the "idiopathic" category) which appear to be suitable for this form of prismotherapy. It must be conceded that this series of cases contains an unusually high proportion of infants and young children with neurological and ocular defects, and this perhaps provides a misleading impression of the limited application of such treatment but even in apparently suitable cases it is doubtful if it is a worthwhile long-term method of treatment.

(2) When the nystagmus is significantly diminished (or sometimes even absent) in a position of convergence, so that there is a significant improvement in the near visual acuity as compared with the distant visual acuity, prisms of appropriate and equal strengths may be placed base-out in front of each eye so that the eyes are retained in the position of convergence and yet permit the binocular fixation of the straight-ahead object in the distance. It is disappointing, however, that in most cases the improvement in the distant visual acuity is less than would be expected from a comparison of the distant and near visual acuities 
before the use of prisms (and in some cases there is no significant improvement), and it is form of therapy which is often only applicable for short periods of time in view of the ey⿳⺈ strain which follow the persistent adoption of a position of convergence.

Furthermore, it is incorrect to assume that the enhancement in the near visual acuity $\overrightarrow{\overline{4}}$ compared with the distant visual acuity which is found frequently in nystagmus inevitab the result of a decrease in the nystagmus in a position of convergence because the enhance ment may occur to an equal extent during a dissociation of the eyes (except when there is $\frac{\text { s. }}{\Phi}$ "latent" element in the nystagmus), and it may occur also in cases of nystagmus in whic one eye is virtually blind; for example, a child in this series with cranio-facial dysostosis an optic atrophy had perception of light in the right eye and $3 / 6$ o and $\mathrm{N}_{5}$ in the left eye with $\overrightarrow{\mathscr{Q}}$ peculiar form of nystagmus which was in an oblique direction. It is difficult, therefore, $\overrightarrow{\mathrm{t}}$ determine the cause of the enhanced near visual acuity in all cases. It is likely that the a禺 of accommodation by itself may exert some influence on the improvement in the visud acuity, quite apart from any alteration of convergence, but it is interesting that, in twes cases of idiopathic nystagmus with a corrected visual acuity of $6 / 60$ and $\mathrm{N}_{5}$ in each ext separately and in both eyes together, this disparity in the distant and near visual acuitis was influenced to only a limited extent by the use of a cyclospastic drug in one eye compared with a cycloplegic drug in the other.

\section{Surgical treatment}

The indications for the application of surgical methods in the treatment of nystagmus are similar to those for the use of the form of prismotherapy which is designed to utilize o position of conjugate gaze in which the nystagmus is least evident. When the nystagmus significantly reduced in a position of conjugate lateral gaze so that there is the adoption an abnormal head posture to achieve this position on looking straight-ahead, an attempit may be made to retain this pattern of reciprocal innervation with the eyes straight-ahea雨. For example, when the nystagmus is least in a position of laevoversion so that the abnorma head posture consists of face turning to the right, a right medial rectus recession and a leff lateral rectus recession alter the position of the eyes so that they become "straight" relation to the face turning to the right. When the face is then turned so that it loo straight-ahead there is a movement of both eyes to the left in order to achieve a straigh ahead position and this is a position in which the eyes have the same innervation as the had in a position of laevoversion before the operation. In this way the position of laev? version (the position of least nystagmus before the operation) is "transferred" to the prima. position of the eyes so that there is no need to adopt an abnormal head posture in order achieve the position of least nystagmus. This approach to the surgical treatment of nystagmus is akin to that of Kestenbaum (1955), who advocated a displacement of the ey towards the quick phase of the nystagmus, that is, towards the direction of the head rotation which turns the eyes into the position of least nystagmus because this induces an impulse turn the eyes in the direction away from the head rotation, and this may be achieved 18 recessions or resections; when the head rotation is to the right the operation may consist $Q$ f right medial rectus and left lateral rectus recessions or right lateral rectus and left medi rectus resections.

The purpose behind the surgical treatment of nystagmus may be regarded in a differe way, although the change in the positions of the eyes is of the same order. When tie "neutral zone" is in a certain position of conjugate gaze (for example, laevoversio recessions of the muscles which are concerned with the slow phase of the nystagmus whien 
turns the eyes into the position of least nystagmus (that is, the right medial rectus and the left lateral rectus) are followed by a conjugate displacement of the eyes to the opposite side (that is, to the right), so that an increased tonus of the muscles which counteract this displacement (that is, the right medial rectus and the left lateral rectus) is necessary, and this is sufficient to achieve a state of equilibrium with the muscles which are concerned with the quick phase of the nystagmus (that is, the right lateral rectus and the left medial rectus), so that the "neutral zone" is transferred to the region of the primary position of the eyes (Anderson, 1953).

It is apparent that these different methods of approach to the direct surgical treatment of nystagmus achieve more or less the same objective and there seems little doubt that this form of treatment provides a better long-term result than prismotherapy. It is unfortunate that parents as a general rule show a reluctance to permit surgical treatment of nystagmus, but this is perhaps a reasonable attitude considering that the advantage of such treatment is essentially the elimination, in whole or in part, of the abnormal head posture which is induced in order to achieve an improved form of visual acuity and not an improvement in the vision as such.

Surgical correction of associated squint

A further application of surgical treatment in nystagmus is the correction of any underlying squint which is present in some cases, and this is of importance because of the enhanced vision which is usually obtained when the eyes function binocularly as compared with uniocularly.

\section{References}

ANDerson, J. R. (1953) Brit. 7. Ophthal., 37, 267

FORD, F. R. (1952) "Diseases of the Nervous System in Infancy, Childhood and Adolescence", 3rd

ed. Thomas, Springfield, Ill.

kestenbaum, A. (I955) "XVII Conc. Ophthal., I954 Canada, U.S.A., Acta", vol. 2, p. IO7 I

KJER, P. (1959) Acta ophthal. (Kbh.), Suppl. 54

WAARdenburg, P. J. (1957) Acta genet. (Basel), 7, 287

WiLson, J. (1967) Proc. roy. Soc. Med., 6o, I 57

windle, w. F. (1950) "Asphyxia neonatorum". Thomas, Springfield, Ill. 